\title{
Análisis comparativo de la logística sostenible entre el Puerto de Veracruz en México y el Puerto de Cartagena de Indias.
}

\author{
Teófilo Omar Boyano ${ }^{1}$ \\ Juan Sebastián Hernández ${ }^{2}$
}

\section{Resumen}

El comercio es la principal actividad económica que jalona el desarrollo económico de las naciones, por ello es de gran importancia el análisis de las capacidades de los puertos, pues son estos en gran medida los responsables de que dicha actividad trascienda la fronteras de las naciones. El objetivo del presente documento es realizar un análisis comparativo que permita comprender la importancia de los puertos de Veracruz en México y Cartagena en Colombia en materia de logística y desarrollo sostenible. Algunos de los hallazgos muestran que, aunque las condiciones morfológicas dan mayor ventaja al puerto de Veracruz, el puerto de Cartagena posee fuertes alianzas estratégicas, lo que favorece al mismo para la ejecución de maniobras logísticas.

\section{Palabras Claves}

Logística sostenible, Desarrollo sostenible, puerto, historia, comercio.

\begin{abstract}
Trade is the main economic activity that marks out the economic development of nations, it is therefore very important the analysis of the capacities of the ports, because they are largely responsible for such activity goes beyond the borders of nations. The objective of this document is to carry out a comparative analysis that allows to understand the importance of the ports of Veracruz in Mexico and Cartagena in Colombia in terms of logistics and sustainable development. Some of the findings show that, although the morphological conditions give greater advantage to the port of Veracruz, the port of Cartagena has strong strategic alliances, which improves itself for the execution of logistical maneuvers.
\end{abstract}

\section{Keywords}

sustainable logistics, sustainable development, port, history, trade.

1. Magister en Administración, docente Institución Tecnológica Colegio Mayor de Bolívar, .

2. Magister en Responsabilidad Social y Sostenibilidad, docente Institución Tecnológica Colegio Mayor de Bolívar. 


\section{Introducción}

En el preámbulo sobre el tema de Desarrollo Sostenible, los países septentrionales europeos hicieron una Convención sobre Desarrollo Humano en el año de 1972, liderado por el Presidente de Estados Unidos, en ese momento histórico Richard Nixon ya que se había presentado un gran impacto ambiental en la Bahía de Santa Bárbara por un derrame de petróleo en el año de 1969. El desarrollo sostenible parte de la apreciación del World Commission on Environment and Development (1987), Our Common Future), conocido como el informe Bruntland que analizó el entorno común, proponiendo más equidad ante la desigualdad de las naciones, luego aparece el Protocolo de Rio en el año de 1992 se insiste en el cuidado del medio ambiente para las generaciones futuras y el cambio climático y posteriormente se estudia el concepto desde el enfoque de la sustentabilidad. Es este último, el concepto como mayor impacto en todos los sectores económico ya que para su aplicación toma a consideración otros conceptos, como es el emprendimiento social el cual ayuda a incrementa el número de empleos $\mathrm{y}$, por consiguiente, disminuye los índices de pobreza y mejora notablemente los índices de calidad de vida, (Cardona, 2017), ejemplo claro de ello se observa en proyectos de emprendimiento adelantados en las comunidades indígenas a través de los cuales se ha podido observar que actividades como el ecoturismo, la agricultura, la confección y las artesanías, son las de mayor acogida por ser estas la que tienen un impacto más notorio en su calidad de vida (Pitre, 2017); por otra parte la sustentabilidad posee un vínculo con la innovación siendo esta un mecanismo novedoso y moderno, adoptado por las pymes, como una herramienta de proyección (Hernández, 2016), dicho vinculo es evidente puesto que la innovación permite la mejor utilización de los recursos, pero como ya se ha indicado esta herramienta se está adoptando y hasta el momento los estudios arrojan que en el sector de las pymes muestra solo que el $40 \%$ de esas empresas desarrollan actividades encaminadas a la innovación (Hernández, 2017).
Dadas las dinámicas que se presentan y la intencionalidad de las Objetivos De Desarrollo Sostenible en una Cumbre en Lima, Perú reuniendo a más de 190 líderes mundiales, redactaron 17 propuestas, o más bien apuestas con la intención de hacer (entre las 17) cada día más ciudades sostenibles; ya que dada la gran aglomeración, los problemas que tiene especialmente en países emergentes y el deseo de hacer un mejor vivir en las ciudades que siguen creciendo, son los criterios que motivan la creación de un estudio comparativo en materia logística entre Veracruz y Cartagena de Indias, dos ciudades afines con vocación portuaria. Veracruz es uno de los principales Estados de Estados Unidos de México y está situada a 400 kilómetros de la Capital o el DF, el Municipio de Veracruz está aledaño al Golfo de México; ya que limita en su parte Este y Norte con ese accidente geográfico. Su temperatura promedio está en $26^{\circ} \mathrm{C}$ y es su Puerto considerado como el principal Puerto Marítimo de México, dado su gran volumen de movimiento de carga. La Ciudad de Veracruz fue descubierta por el adelantado Juan de Grijalva, pero fundado por Hernán Cortes en el año de 1519, vieron su potencialidad para movilizar mercancías y se convertiría en un Pueblo de carácter marítimo muy significativo para la Corona Española, por su zona logística natural. Entre sus sitios turísticos se encuentra la Catedral, las Plazas Municipal y la Plaza Mayor, el Teatro de la Reforma y el famoso faro Venestuniano Carranza; La Ermita del Rosario en la Veracruz vieja y el Faro de Benito Juárez. Además, en su área metropolitana cuenta con los grandes Fuertes de San Juan de Ulúa, construido sobre una Isla al frente de Veracruz. El Baluarte de Santiago es otro reconocido sitio turístico, dichos Fuertes fueron construidos en el Siglo XVI para defenderse de los piratas. Entre sus principales balnearios están: Costa de Oro, Mocambo, Playón de Hornos y un gran Arrecife denominado el Sistema Arrecifal Veracruzano y el tradicional Malecón de Veracruz. Además del World Trade Center de Veracruz para eventos y grandes convenciones y el concurrido Plaza de las Américas su principal Centro Comercial. 
Figura 1. Fuerte de San Juan de Ulúa

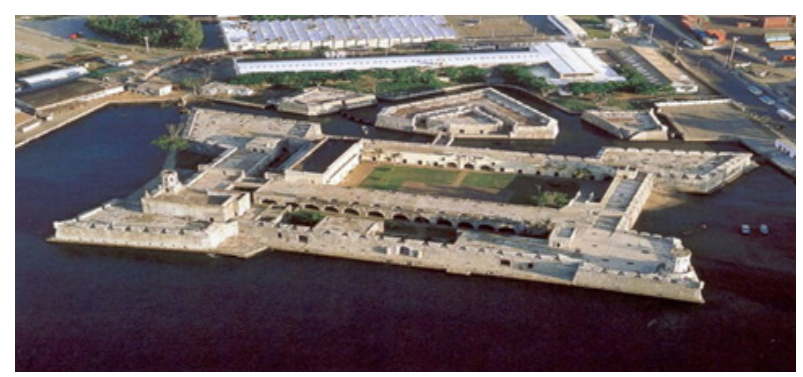

Fuente: www.toursstore.com

Este Fuerte fue primero una prisión y recuerda para el caso de Colombia a Gorgona en cuanto a su percepción, no en cuanto a su georreferenciación, ya que Gorgona es una Isla bastante distante de Plataforma continental, mientras que el de San Juan de Ulúa está muy cerca y fue construido artificialmente. Construido en el año de 1535 por Juan de Mendoza y finalizado casi 172 años después. El sitio también fue utilizado como almacenamiento de metales preciosos y de desembarco. El Puerto de Veracruz comienza a tener gran actividad cuando según Domínguez Pérez, O. (Pág. 89. 1990) sostiene que "en el año de 1873 el día 1 de enero arriba a la ciudad proveniente de ciudad de México el ferrocarril, era esperado por el presidente Sebastián Lerdo de Tejada". La compañía inglesa hizo que llegara a la ciudad amurallada de Veracruz el empuje del comercio y la conectividad.

\section{Figura 2. Plano Topográfico de Veracruz}

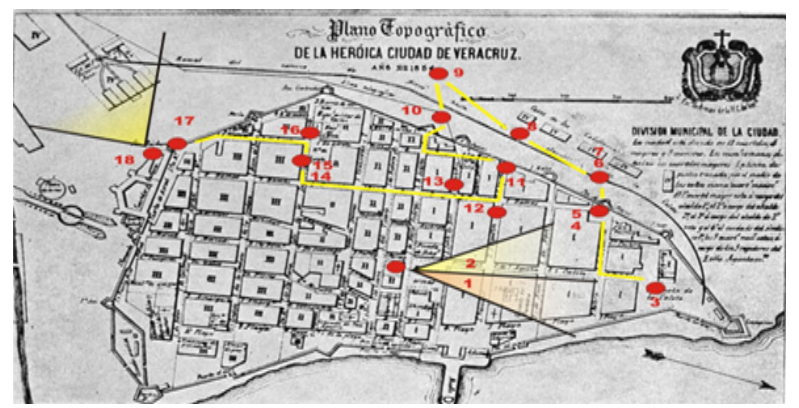

Fuente: aguapasada.wordpress.com

Nótese la gran similitud de la ciudad en ese momento histórico con el llamado Corralito de Piedra Cartagena y lleva el mismo título de Heroica, para el caso de Veracruz por haber defendido desde el Fuerte más de cinco ataques

Ediciones EFIM hasta el año de 1538 cuando terminó la última confrontación con los españoles. Cartagena de Indias por el contrario no solo tuvo ataques de españoles, si no que bajo el poderío de la conquista española tuvo ataques de Piratas que la quemaron y saquearon, algunos llevan el nombre honorífico de Sir por la Corona inglesa. En cuanto a Cartagena es una ciudad pletórica en leyendas y datos históricos corroborables, fundada en 1533 luego de vencer a los Kalamarí, Pedro de Heredia la funda un 1 de junio dado el punto estratégico logístico que tenía su Bahía segura y propicia para llevar metales preciosos y mercancías hacia España, de allí recibió el nombre de Cartagena de Indias. Las grandes fortificaciones que presenta el Distrito de Cartagena, fueron diseñadas y construidas en gran parte por Antonio de Arévalo, sus Murallas y Baluartes sirvieron también para la defensa de los Piratas y Corsarios. Son muy visitados las Baterías de San Felipe de Barajas, San Fernando y San José que separan la entrada a la Bahía por una boca pequeña que se le llamó Bocachica. Entre otras sus gestas de Independencia y su deseo de crecimiento durante el Siglo XX, a orillas del Caribe, con temperaturas que oscilan entre $20{ }^{\circ} \mathrm{C}$ a $32^{\circ} \mathrm{C}$ y mayormente con gran humedad, excepto por la llegada de los vientos alisios a principios de año que coinciden con la alta temporada turística. La Heroica como también se le conoce a Cartagena llegaría el ferrocarril años después, como sostiene Meisel-Roca, A. (Pág. 3, 2015) señala que "siendo el 1 de agosto de 1894 con el tren de la Ruta Calamar Cartagena", la ciudad había dejado atrás el gran receso económico y demográfico ocasionado por su Independencia y el sitio de Morillo comisionado por la Corona Española que el 5 de diciembre de 1815 había abierto sus puertas después de 115 días de cierre forzado y encontró más de la mitad de la población muerta y el resto enferma y hambrienta, por esa razón la ciudad tardó más de 55 años en iniciar su recuperación".

La Ruta Calamar- Cartagena tenía un recorrido de más de 105 kilómetros de distancia, con 11 estaciones, 85 vagones y con 4 locomotoras. Trajo dinámicas interesantes de comercio y la ciudad reinició su crecimiento 
varios años después saliendo del Centro Amurallado y con gran cantidad de migrantes, entre los que se destacan los Sirio-libaneses.

\section{Figura 3. MUHCA}

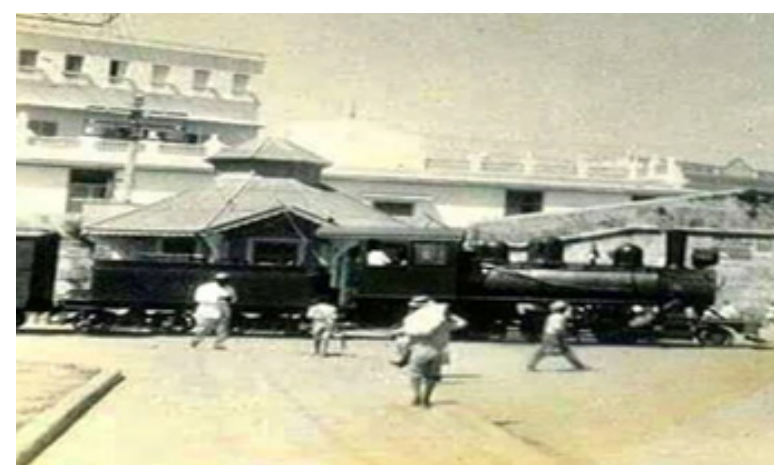

Fuente: www.eluniversal.com.co

En el caso de Cartagena de Indias desde un estudio que revela la asimetría de los Puertos de Colombia, lo presenta un equipo de investigadores entre los que se encuentra Castell, O. C. (2017) señalan que Las dinámicas económicas de las décadas de los ochenta y noventa, junto a lo que ha transcurrido del siglo XXI, han configurado nuevos intereses y relaciones comerciales, impactando, entre otras cosas, las rutas del comercio marítimo, (pág. 88) y 1levando de igual modo a el puerto de Cartagena a desarrollar competencias diferenciadoras y necesarias para garantizar la sostenibilidad y competitividad, lo que sea considerado una estrategia innovadora (Hernández, 2017). En cuanto al concepto de logística y su relación con el concepto de ciudad, los primeros datos encontrados, no necesariamente cronológicamente con la temática están en la Red de Observatorios Nacionales, que realiza El Banco Interamericano de Desarrollo, dicho Observatorio, tiene en cuenta la Logística sostenible que busca reducir el impacto de la logística con el desarrollo y el crecimiento de las actividades comerciales. El concepto de Logística dejó de ser enfocado a cuestiones de ahorro de dinero, y se volvió más relevante el impacto ambiental que genera las actividades de aprovisionamiento y la cadena de suministro. Hoy día se habla también de la logística urbana, también reconocida como logística de la última milla, incluye todos movimientos comerciales y el aprovisionamiento que incluye el suministro y la manera de cómo se distribuye en la ciudad. (BID, 2018) Como aspecto importante relacionado a la ciudades y puertos sostenibles, esfuerzos interesantes se han hecho por El Puerto de Cartagena y Contecar que unidos forman la gran Sociedad Portuaria y uno de los más grandes proyectos que ha incrementado el tránsito de contenedores ha hecho un acuerdo con la Financiera de Desarrollo Territorial. Findeter y dentro del marco de estudios que proyectan a las ciudades sostenibles el 10 de febrero de 2017 y registrado por Grupo Puerto de Cartagena (2017) en dicho escenario se comprometieron a reducir su impacto ambiental, ya que ha sido el gran tópico que se exigió en la XXI Conferencia Internacional sobre Cambio Climático, hecho en París durante el año 2015 y con la esperanza de reducir la emisión de gases para el 2030 en un 20\%. Fuentes, D. D. (2014) Sin lugar a dudas los sistemas de transportes y logística han estado estrechamente relacionados a las transformaciones históricas en el comercio, la inversión y la movilidad del capital humano a escala mundial (pág., 20) es un tema interesante que abarca el estudio del Puerto de Cartagena y el ensanche de la infraestructura portuaria en Cartagena y el Caribe.

\section{La Logística y el desarrollo sostenible un estudio comparativo}

Un estudio relacionado con el Patrimonio dentro del territorio y su relación con el desarrollo sosteniblehechoporToribio, J.M.F.(2010) señalaque: El artículo plantea explorar, a través del análisis comparativo de cinco ámbitos de estudio en Iberoamérica y España, las relaciones entre patrimonio territorial y desarrollo sostenible. Para cubrir dicho objetivo, es necesario profundizar en las bases conceptuales y metodológicas que implican ambas nociones y, a partir de ellas, realizar un trabajo de sistematización empírica que permita reconocer y evaluar, sobre patrones comunes, las características del patrimonio territorial y su relación con el desarrollo sostenible en cada uno de los ámbitos; las conclusiones derivadas del análisis son ambivalentes, ya que a la demostrada riqueza del patrimonio territorial en todos los ámbitos analizados y a su mejor situación relativa en cuanto a desarrollo socioeconómico, se une una desequilibrada explotación de los recursos territoriales y una escasa atención Anfibios ISSN: 2665-1513 
a las cuestiones de sostenibilidad ambiental. El anterior estudio evidencia que no hay mucha claridad relacionada con el tema de sostenibilidad, desarrollo económico y el territorio; mientras que otro estudio comparativo de ciudades sostenibles entre Sao Paulo y Bogotá relacionado con tecnologías y certificaciones de construcción, hecho por González Lizcano, K. L. (2017). sostienen que: Se presenta una comparación de las iniciativas y proyectos de construcción sostenible entre las ciudades de Sao Paulo-Brasil y Bogotá- Colombia. Se hace una evaluación de las metodologías aplicada (certificaciones), el conocimiento de cada una y de los avances al respecto en cada ciudad. Como insumo principal, se realizó una visita a la universidad de Sao Paulo, Brasil, a partir de esta información, se realizó una evaluación y comparación de los principales sellos y certificaciones de esta ciudad. Posteriormente se recolecto la misma información en la ciudad de Bogotá, finalmente se analizó y comparo la situación de la de la construcción sostenible, en las dos ciudades, con el fin de hacer un diagnóstico de los aportes al desarrollo sostenible urbano. El anterior estudio hecho en dos grandes ciudades o aglomeraciones urbanas, muestra el interés de conservar el desarrollo sostenible y que mejor hacerlo que en Sao Paulo que fue durante varios años la capital de Brasil, y la capital de la República de Colombia, Bogotá que ha tenido serios problemas de emisión de gases; otro estudio de caso hecho propiamente en Cartagena y relacionado con la competitividad y políticas para el desarrollo sostenible, presentado por López, J. A. (2018) señala que: Mediante un análisis crítico relacionado con dificultades conceptuales y empíricas de políticas públicas y el desarrollo de ciudades sustentables, en el año 2015 para Cartagena de Indias, Colombia, delimitando componentes afines y definidos en la medición de competitividad en ciudades-puerto e intermedias en Latinoamérica, se hizo mediante interpretación y análisis en tres enfoques que fueron: local, nacionales e internacionales: la Iniciativa Ciudades Emergentes y Sostenibles (ICES), del Banco Interamericano de Desarrollo. BID; el Programa Cidades Sustentáveis y su guía GPS Gestão Pública Sustentável y la norma ISO 37120 que enmarca al Desarrollo Sostenible en ComuniEdiciones EFIM dades (Indicadores para servicios de la ciudad y calidad de vida). Se presupone coherente y con jerarquía sobre las políticas públicas para las ciudades; aunque, se revisan reflexiones de actores sociales y expertos participantes en talleres de análisis de políticas públicas, al tiempo que se evalúa ideas discursivas en materia de competitividad cuando se establecen políticas de desarrollo territorial y se observa sus efectos en la ciudad estudiada; se exponen dificultades, los hallazgos y las consideraciones metodológicas y prácticas que se revelaron al intentar este ejercicio de consenso. Dicho ejercicio estuvo sustentado en análisis sistémico del comportamiento, influencia y potencial de desarrollo de cada subcomponente y tema clave del sistema territorial de Cartagena de Indias, por último, deja la reflexión sobre aportes de este caso, en cuanto a proceso y resultados, pertinente para ciudades-puerto de Latinoamérica en búsqueda de sustentabilidad y la competitividad. El anterior estudio busca el imaginario de personas versadas, además de las tres instancias que depuran la información, como son el Puerto o localidad, a nivel nacional e internacional y llama la atención n que el estudio se hace con la Norma ISO 37120, circunscrito a Ciudades Sostenibles; otros estudios relacionados con el componente logístico ya relacionado a la logística interna aplicado a los Centros de Distribución realizado por Montoya, R. A. G. (2018). sostienen que:

Mediante un desarrollo y su validación de un modelo de centro de distribución (CEDI) verde, que se conforma de tres componentes: un módulo de gestión, un sistema de administración de almacenes (WMS-Warehouse Management System) y un metaheurístico. Como resultado de la validación del modelo en un CEDI de una empresa mediana de alimentos, se obtuvo una reducción de las emisiones de $\mathrm{CO} 2$ equivalente de $731 \mathrm{~kg} / \mathrm{mes}$ o un $37 \%$ de las emisiones en la preparación de pedidos. Además, se desarrolló el modelamiento de un metaheurístico de búsqueda tabú para resolver el problema de ruteo en la preparación de pedidos, lo que permitió incrementar la eficiencia de la operación en un $18,83 \%$, así como obtener una reducción de $198 \mathrm{~kg}$ de CO2 equivalente por mes. Por lo tanto, el modelo CEDI verde, 
simultáneamente, aumentó la eficiencia y redujo la cantidad de kilogramos de $\mathrm{CO} 2$, utilizando un enfoque que no había sido abordado en la literatura para este proceso logístico. Sigue siendo relevante el tema de emisión de gases en las actividades logísticas, ya que en las maniobras también se utiliza el transporte; como lo resalta en su libro Políticas Integradas de Infraestructura, Transporte y Logística; experiencias internacionales y propuestas iniciales, una investigación hecha por su equipo de trabajo, Cipoletta Tomassian, G. (2010) señalan que: "Las políticas públicas que se relacionan con el transporte y la infraestructura no se han analizado en conjunto y sostiene que la logística no ha sido tratada de manera ideal porque no ha sido sistemática y orgánica su forma de abordar; además aduce que eso ha ocasionado ineficiencia en el servicio en lo relacionado a la infraestructura, al tiempo que no ha permitido el desarrollo sostenible en el Caribe y en Latinoamérica, sostiene además que, la situación se complica por la ausencia de políticas de gobierno relacionadas al tema de infraestructura, transporte y la logística como una gran herramienta estratégica y competitiva según el autor",

\section{La Logística del turismo factor clave para la competitividad.}

Las ciudades Portuarias de Veracruz y Cartagena deben mantener o motivar al liderazgo en el proceso de la logística no solo de carga, sino también de pasajeros y aunque sigue siendo un gran generador de divisas para los dos Puertos, el reto es que cada vez más mejore la atención, eficiente y oportuna ante el escaso tiempo que demora un buque de cruceros en las ciudades turístico portuarias. Para citar un caso; en Cartagena de Indias hay un estudio relacionado con la percepción de usuarios en el servicio de cruceros en el Puerto de Cartagena, en el cual un equipo de investigadores con Juan Gabriel Brida (2012) que en un estudio amplio con una gran muestra de pasajeros sostuvieron: El propósito de este trabajo es explicar la satisfacción en tierra de los pasajeros de cruceros en el puerto de escala de Cartagena de Indias. Se hizo metodológicamente, así: se entregó un cuestionario a 1.361 pasajeros de 28 cruceros durante octubre y noviembre de 2009 , luego se emplearon análisis de factores y análisis de conglomerados. Los resultados encontrados fueron: A pesar que, aunque los visitantes mantuvieron una alta satisfacción general de la experiencia en tierra, hay dos dimensiones que requieren la atención de los responsables de la política turística: la infraestructura de la ciudad (tráfico, ruido, limpieza e infraestructura) y la experiencia general de compra. En particular, la peor experiencia parece estar relacionada con los vendedores ambulantes. Además, hay evidencia de que los turistas de EE. UU. Son más exigentes de estar completamente satisfechos. Por lo que no solo se trata de Infraestructura Logística, también de los usuarios de los puertos y ciudades de gran aglomeración, específicamente otra investigación ya relacionado con el Puerto de Veracruz; presenta un estudio de carácter holístico que involucra la convergencia dentro de las regiones y el crecimiento relacionado con el Puerto de Veracruz, Estado de México y liderado por Gregory J. Brock. (2014) el cual sostuvo que: ¿La experiencia mexicana de crecimiento y convergencia interregional también se produjo en regiones únicas? Usando la importante región sureña de Veracruz, el propósito de este documento es examinar esta cuestión en un período de 48 años dentro de un único estado mexicano. Se hizo metodológicamente así: El crecimiento se examina utilizando una función estándar de producción estocástica de dos entradas (SPF) que crea una medida de eficiencia técnica. La convergencia se mide usando una ecuación de convergencia de la literatura, pero que también incluyó los resultados del análisis SPF para incorporar no solo los niveles iniciales de insumos, sino también la capacidad de un municipio para utilizar estos insumos. La recolección de datos en México y en línea incluyó una base de datos a largo plazo de 149 municipios en Veracruz de 1960 a 2008. Con los siguientes hallazgos: una tecnología estocástica de Cobb-Douglas encaja bien en el crecimiento a largo plazo de la provincia de Veracruz. En la década de 1960, 2000 y el largo plazo (19602008), la evidencia débil para los municipios de Veracruz parece estar convergiendo con un nivel relativamente más alto de eficiencia técnica que resulta en un crecimiento más Anfibios ISSN: 2665-1513 
lento de la productividad del trabajo industrial. Algunas mejoras muy recientes en la eficiencia técnica pueden ser el resultado de reformas institucionales y económicas que finalmente permiten la salida de empresas ineficientes que han mantenido los niveles de eficiencia técnica industrial del municipio estancados durante décadas en aproximadamente 70 por ciento. Aspectos claves como el crecimiento de la Industria y el Puerto son temas interesantes para tener en cuenta en el Puerto de Cartagena que también tiene la Zona Industrial de Mamonal, aunque varias de sus empresas están relacionadas con el sector logístico portuario. 4. ¿Son Veracruz y Cartagena Puertos Pivotes? Los Puertos Hub o Concentradores se les denomina pivotes, porque son considerados mayores, estén localizados en país, región o cualquier pueblo pequeño, su mayor actividad es el tráfico de contenedores y tiene la particularidad de ser suplidos por Puertos menores o alimentadores y se concentran en esa actividad y es tal el volumen de cargas que movilizan que son operados por buques que transportan gran volumen de contenedores llamados buques Postpanamax. No puede confundirse con un mega puertos que es considerado un Sistema de Puertos, el Puerto Pivote hace parte de ese Sistema. Pero si se va la Definición de mega puerto que incluye grandes volúmenes de movilidad de mercancía contenedorizada y al granel, a su vez que tienen varios Puertos que hacen diferentes tipos de actividades, se pueden considerar a los Puertos de Veracruz y de Cartagena como mega puertos y no como Puertos Pivotes.

\section{Figura 4 Ubicación Geográfica del Estado de Veracruz.}

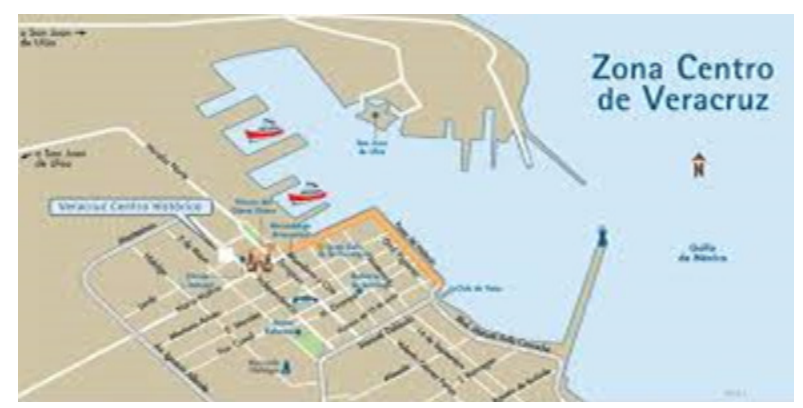

Fuente: war-thunder-sajt.ru

Nótese la Zona Portuaria en gran parte por el crecimiento artificial del Puerto de VeraEdiciones EFIM cruz que ha permitido mejora las maniobras logísticas portuarias, al tiempo que va unido a el cuidado de su infraestructura vial.

El Estado de Veracruz tiene una envidiable posición logística, su capital es Xalapa que se halla a 90 kilómetros de la ciudad portuaria de Veracruz: junto al Puerto de Altamira están ubicados en el gran Golfo de México; mientras que, el Puerto de Cartagena en la parte Norte de Suramérica y en sitio estratégico que le permite ser muelle de transbordo, ya que por su Bahía segura y con pocas posibilidades de impacto directo de huracanes la hace apetecida para maniobras logísticas, El Estado de Veracruz posee el $37 \%$ de la franja costera de México y con una franja costera de 684 kilómetros y está al Este del Golfo de México, con 10 Regiones y agrupadas en 212 municipios, y una población que ronda los 7, 2 millones de habitantes incluyendo su área metropolitana, además que el $35 \%$ de las vertientes hidrográficas hacen parte de Veracruz y desembocan en el Golfo de México, el Estado tiene ocho ciudades llamadas medias por su número de habitantes y extensión, entre las que se encuentran: Poza Rica Xalapa, Orizaba, Boca del Río, Córdoba, Minatitlán, además de la ciudad de Coatzacoalcos. Datos históricos, relacionados con la fundación y la posibilidad de el gran impacto del mestizaje por parte de los africanos que llegaron en tiempos de la conquista, del Puerto de Veracruz, se presenta por Rinaudo, C. (2012) quien sostiene que: en cuanto a la llegada de África al Puerto de Veracruz, no es marcada la influencia y si bien es cierto que llegaron, en la actualidad la cultura afrodescendiente no es marcada o al menos no reivindicada, sin embargo, se evidencia la existencia en Oaxaca y Coyolillo (pág. 12) Fruto de ese mestizaje se dio el termino de jarocho al mexicano, alegre y espontáneo que vive en las Playas, a lo que se le denomina en Colombia costeño. La Unesco (1970) hizo un texto sobre "la ruta del esclavo en los años 90". Mientras que en la fundación del Puerto de Cartagena si es muy marcada la presencia e influencia de los africanos que dejaron su huella en las grandes construcciones de sus Fortificaciones y Baluartes, al igual de la influencia de Pedro Claver, llegado a ser reconocido como Santo o el Apóstol de los Negros.

El barrio de Getsemaní y su Bahía de las 25 
Ánimas fue la zona por donde se daba gran parte de las maniobras logísticas y permitió durante muchos años, más de IV Siglos el movimiento de mercancías y posteriormente las grandes actividades comerciales.

\section{Figura 5 Ubicación geográfica del Puerto de Cartagena.}

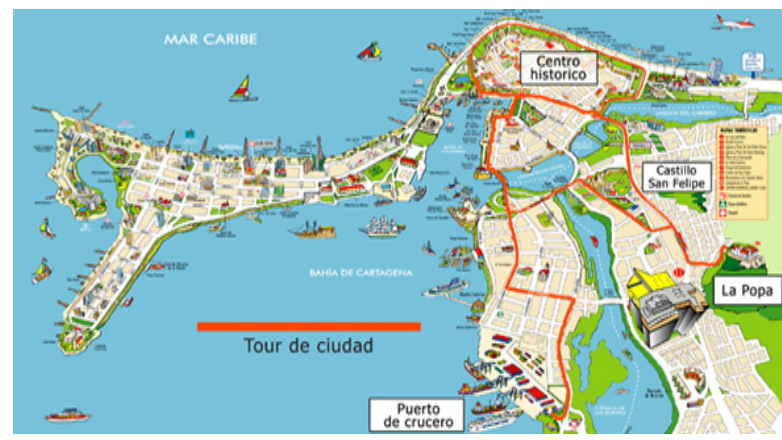

Fuente: pinceladas-ilp.com

Nótese la imagen que muestra el paisaje natural y turístico unido a las facilidades naturales que le da la morfología del Puerto para hacer las maniobras logísticas portuarias.

Como en todas las ciudades portuarias, al momento de incrementarse el volumen de carga o mercancías movilizadas, generar problemas de movilidad y de sostenibilidad, para el caso de Veracruz, hay un estudio liderado por Fuentes Campos, R. (2018) señala que: Actualmente, el movimiento de carga en el recinto portuario presenta un incremento en la tasa media de crecimiento anual del $16,4 \%$ y en la presente década se han observado preocupantes signos de congestionamiento. Además, constantemente aparecen nuevas tecnologías de transporte de mercancía, las cuales se ven reflejadas en buques de mayores dimensiones que ya no pueden ser atendidos en el puerto actual por las limitaciones de su configuración, concretamente por cuestiones de insuficiencia de calado, de posiciones de atraque y de superficies de almacenamiento. (pág. 6)
Tabla 1: Datos Demográficos e históricos comparativos de los dos Puertos

\begin{tabular}{|c|c|c|}
\hline Municipio & Veracruz & $\begin{array}{c}\text { Cartagena de } \\
\text { Indias }\end{array}$ \\
\hline $\begin{array}{l}\text { Población incluyen- } \\
\text { do Zona Metropoli- } \\
\text { tana a } 2015\end{array}$ & $\begin{array}{l}\text { Municipio } 475.000 \\
\text { Zona Metropolitana } \\
811.671\end{array}$ & $\begin{array}{l}\text { 1,001.076 incluyen- } \\
\text { do Zona Metropo- } \\
\text { litana }\end{array}$ \\
\hline $\begin{array}{l}\text { Tasa de crecimiento } \\
\text { poblacional }\end{array}$ & $1 \%$ & $1,5 \%$ \\
\hline Superficie & $241 \mathrm{Km} 2$ & $609.1 \mathrm{Km} 2$ \\
\hline $\begin{array}{l}\text { Densidad pobla- } \\
\text { cional }\end{array}$ & $\begin{array}{l}1800 \mathrm{hab} / \mathrm{Km} 2 \\
\text { Aprox. }\end{array}$ & $1.811,91 \mathrm{hab} / \mathrm{Km} 2$ \\
\hline $\begin{array}{l}\text { Estado o Departa- } \\
\text { mento }\end{array}$ & $\begin{array}{l}\text { Veracruz de Ignacio } \\
\text { de la Llave }\end{array}$ & Bolívar \\
\hline $\begin{array}{l}\text { Año de Fundación y } \\
\text { Nombre }\end{array}$ & $\begin{array}{l}1519 \text { Villa Rica de la } \\
\text { Vera Cruz }\end{array}$ & 1533 \\
\hline Fundador & Hernán Cortes & Pedro de Heredia \\
\hline Títulos & $\begin{array}{l}\text { Ciudad Patrimonio } \\
\text { de la Humanidad }\end{array}$ & $\begin{array}{l}\text { Ciudad Patrimonio } \\
\text { de la Humanidad }\end{array}$ \\
\hline Afinidad & $\begin{array}{l}\text { Ciudad hermana de } \\
\text { Cartagena de Indias }\end{array}$ & $\begin{array}{l}\text { Ciudad hermana de } \\
\text { Veracruz }\end{array}$ \\
\hline Desempleo & Inferior al $5 \%$ & Superior al $10 \%$ \\
\hline Infraestructura vial & $\begin{array}{l}\text { Bien trazada gracias } \\
\text { a morfología natural } \\
\text { con más de } 1.000 \\
\text { kilómetros en vías }\end{array}$ & $\begin{array}{l}\text { Problemas al trazarla } \\
\text { debido a que es un } \\
\text { archipiélago natural }\end{array}$ \\
\hline $\begin{array}{l}\text { Proyección infraes- } \\
\text { tructura portuaria }\end{array}$ & $\begin{array}{l}\text { Gran inversión y } \\
\text { aumento de sitios } \\
\text { logísticos portuarios }\end{array}$ & $\begin{array}{l}\text { Inversión y creci- } \\
\text { miento }\end{array}$ \\
\hline Aeropuertos & $\begin{array}{l}\text { De gran movilidad el } \\
3 \text { en México }\end{array}$ & $\begin{array}{l}\text { De gran movili- } \\
\text { dad es el } 4 \text { a nivel } \\
\text { nacional }\end{array}$ \\
\hline $\begin{array}{l}\text { Geoecología del } \\
\text { paisaje }\end{array}$ & $\begin{array}{l}\text { Problemas reducidos } \\
\text { con los palaperos }\end{array}$ & $\begin{array}{l}\text { Algunos incidentes } \\
\text { aislados con mala } \\
\text { atención }\end{array}$ \\
\hline $\begin{array}{l}\text { Administración } \\
\text { Pública }\end{array}$ & $\begin{array}{l}\text { Gran liderazgo con } \\
\text { Adolfo Ruiz Corti- } \\
\text { nes quien fue Presi- } \\
\text { dente de México y } \\
\text { gobierno estable a } \\
\text { nivel municipal }\end{array}$ & $\begin{array}{l}\text { Pérdida de liderazgo } \\
\text { en los últimos } 20 \\
\text { años. Aunque Rafael } \\
\text { Núñez ha sido el } \\
\text { Presidente que más } \\
\text { veces ha sido presi- } \\
\text { dente en Colombia. }\end{array}$ \\
\hline Fiestas tradicionales & Carnaval Veracruz & $\begin{array}{l}\text { Fiestas de la Inde- } \\
\text { pendencia }\end{array}$ \\
\hline $\begin{array}{l}\text { Otras actividades } \\
\text { culturales }\end{array}$ & $\begin{array}{l}\text { Navideñas, y Sema- } \\
\text { na Santa y Regata } \\
\text { amigos de Galveston }\end{array}$ & $\begin{array}{l}\text { Rica en actividades } \\
\text { musicales, festivales } \\
\text { y eventos como } \\
\text { Hay Festival, El del } \\
\text { Dulce, del Frito y de } \\
\text { música, entre otros }\end{array}$ \\
\hline
\end{tabular}

Fuente: Elaboración propia

Los Puertos tienen una gran diferencia en extensión siendo Veracruz mucho más pequeño, tres veces menos grande, pero con una densidad poblacional casi semejante, mientras que en Cartagena de Indias hay mucha informalidad en el empleo y pobreza, en el Puerto de Veracruz es menor y hay políticas públicas de inclusión la- 
boral, las dos localidades fueron fundadas por la Corona Española a inicios y durante el Siglo XVI. Se considera el Puerto de Veracruz como el más importante para el comercio de México, el Puerto de Cartagena también lo es desde el punto de vista del comercio, ya que, para el caso de Veracruz, por casi dos Siglos y medio fue el único Puerto de México por donde entraba y salía mercancías hacia el Viejo continente o Europa. El Puerto de Cartagena es el de mayor movimiento en contenedores en Colombia y en el turismo de cruceros. La unión con Contecar ha generado una dinámica bien interesante que proyecta a su Puerto como uno de los más importantes de Suramérica después de Santos en Brasil. En cuanto a la movilidad urbana, los comunes a nivel de Latinoamérica padecen los mismos problemas de: infraestructura vial acorde con la población, la calidad del transporte público, desafíos ambientales y gestión para la regulación. Es más notorio el problema de movilidad en el Puerto de Cartagena. No debe confundirse el Municipio de Veracruz con el Estado de Veracruz ya que sus condiciones son muy diferentes, mientras el Estado en general padece problemas de algunos brotes de violencia, el Municipio gracias a su generación de empleo y alto nivel de educación como política pública su nivel de violencia es muy bajo. A nivel de la percepción relacionada con el espíritu empresarial y en cuanto a las dinámicas urbanas, en México, Torres Tello, Giovanna Patricia (2018) señala que. $\mathrm{Su}$ estudio se encuentra centrado en la reconstrucción de estrategias empresariales de expansión principalmente, y que son tomadas como acciones realizadas por el empresario. El enfoque con el cual analizaron la implementación de las estrategias empresariales es a partir de que existen diversos factores estructurales y culturales, así como diversas redes sociales que se mueven en diferentes niveles (familia, amistad, compadrazgo, étnico, empresarial, político) que las van configurando a partir de que el empresario es visto como sujeto social en interacción, donde a partir de su subjetividad del sujeto de sus acciones le dan sentido a su actividad empresarial. Sin embargo, no se niega la existencia en el empresario de la búsqueda de la máxima ganancia y de beneficio, además de una racionalidad al hacer negocios, sino que además existe Ediciones EFIM en el empresario procesos de mediación subjetivos y culturales que le dan sentido a una situación concreta. Asimismo, se explica la polémica de si el empresario debe ser concebido como actor racional en donde siempre se encuentra en la búsqueda de máxima ganancia, teniendo toda la información para calcular sus acciones y obtener soluciones óptimas. Sin embargo, esta posición de analizar al empresario parece un tanto limitada, porque no da cuenta de una diversidad de factores que forman parte de una realidad compleja en donde entra en juego para la explicación y comprensión de las acciones empresariales.

En cuanto específicamente el Puerto de Veracruz, Barbero, J. (2011) señalan que Veracruz tiene un incremento del $5 \%$ en su parque automotor en los últimos 20 años, y en cuanto a los kilómetros por vehículo recorrido está en $8.8 \%$ muy por encima de gran parte de las otras principales ciudades del México. En cuanto al ensanche de la Infraestructura portuaria hay estudios en ambas ciudades relacionados con el tema, para el caso de Cartagena de Indias se pretende adecuar el sitio de la Escollera de la Marina, donde se cree que se hizo un bajo artificial para que no entrasen los piratas a la ciudad y encallasen las embarcaciones en tiempo de la conquista española, dicho paso sería el sitio por donde pasaría el Canal del Varadero, su construcción está en entredicho, se aduce impacto ambiental y posible daño en infraestructura turística urbana, específicamente edificios del Barrio El Laguito. Estudios relacionados con la interconectividad e infraestructura, presentan a Fuentes Campos, R. (2018) quien sostiene que: "para el caso de México se pone como meta porque es necesario establecer las necesidades y a nivel macroeconómico si se amplía para la zona norte conectaría el subsector de servicios ferroviarios y con la infraestructura vial existente, al igual que las Zonas de Actividades Logísticas y el terminal de carga aérea y jalonando el sector industrial". En cuanto a la infraestructura urbana, Veracruz tiene grandes arterias por donde se mueve gran parte de su población y son:

Rafael Cuervo- Cuauhtémoc

Allende- Miguel Alemán

Díaz Mirón 
Las vías están construidas de 6 a 10 carriles y van en doble sentido, seis grandes puentes, el más antiguo se llama Morelos, con dos grandes distribuidores viales que son: J. B. Lobos que llega a su Aeropuerto llamado Heriberto Jara Corona el tercer más importante y concurrido de México, y el segundo distribuidor vial es llamado Olmeca, en honor a sus antepasados indígenas. Tiene además un Sistema de transporte terrestre de pasajeros denominado Autobuses de Oriente. ADO

De acuerdo a datos encontrados relacionados con su movimiento y tendencia del Puerto, se puede anotar que: (Coordinación General de Puertos, 2018) según la Secretaría de Comunicaciones y Transporte. SCT, "Existen tres Puertos importantes en México y son el Puerto de Manzanillo, el Lázaro Cárdenas y el de Veracruz.

México tiene 117 Puertos y Terminales habilitados que superan los 300 millones de toneladas movilizadas. Con dos Océanos y posición logística óptima por ser vecino de Estados Unidos y con gran infraestructura portuaria.

Se estima que al terminar el 2018 México movilizará más de 66 millones de toneladas y que para el 2024 alcance 90 millones de toneladas movilizadas" La Secretaría de Comunicaciones y Transporte tiene el compromiso de llevar a cabo esa mega obra dentro del marco del Programa Nacional de Infraestructura. PNI, con una inversión de 30.000 millones de pesos mexicanos y con el $80 \%$ de inversión público- privada, dando mayor garantía al proceso.

El Puerto de Veracruz movilizó en el año 2017 un aproximado de 27.8 millones de Toneladas, y en cuanto al número de contendores movilizados sobrepasó el millón de contenedores, es decir 1.117 Teus con alrededor de un millón treinta y un mil autos movilizados, el saldo en mercancía suelta, fluidos, hidrocarburos, graneles agrícolas y minerales.

Tiene el Municipio de Veracruz una Capitanía de Puerto que vigila 8 Muelles, del Puerto de Veracruz, los terminales de Icave, Pemex, Terminal Turística.
Tabla 2: Agencias Marítimas y Líneas Navieras que ofrecen servicios de carga contenedorizada en el Puerto de Veracruz son.

\begin{tabular}{|l|l|}
\hline \multicolumn{1}{|c|}{ Líneas Navieras } & \multicolumn{1}{|c|}{ Agencia Marítima } \\
\hline Hapag Lloyd & $\begin{array}{l}\text { HAPAG-LLOYD MÉXICO, } \\
\text { S.A. DE CV }\end{array}$ \\
\hline Melfi Marine Co. S.A. & $\begin{array}{l}\text { AGENCIA NAVIERA DE MÉ- } \\
\text { XICO S.A. DE C.V }\end{array}$ \\
\hline Hiundai Merchant Marine & $\begin{array}{l}\text { NORTON LILLY SHIPPING } \\
\text { MEXICO S.A. DE C.V. }\end{array}$ \\
\hline ZIM Container Lines & $\begin{array}{l}\text { ALTAMARÍTIMA, S.A. DE } \\
\text { C.V. }\end{array}$ \\
\hline $\begin{array}{l}\text { CMA CGM México S. A. DE } \\
\text { C. V. }\end{array}$ & $\begin{array}{l}\text { CMA CGM México S. A. DE } \\
\text { C. V. }\end{array}$ \\
\hline $\begin{array}{l}\text { HAMBURG SÜD SHIPPING } \\
\text { GROUP }\end{array}$ & $\begin{array}{l}\text { HAMBURG SÜD MÉXICO, } \\
\text { S.A. DE C.V. A }\end{array}$ \\
\hline ALIANÇA & $\begin{array}{l}\text { HAMBURG SÜD MÉXICO, } \\
\text { S.A. DE C.V. A }\end{array}$ \\
\hline $\begin{array}{l}\text { CIA CHILENA DE NAVEGA- } \\
\text { CIÓN INTEROCEÁNICA S.A. } \\
\text { (CCNI) }\end{array}$ & $\begin{array}{l}\text { HAMBURG SÜD MÉXICO, } \\
\text { S.A. DE C.V. A }\end{array}$ \\
\hline MAERSK LINE & $\begin{array}{l}\text { MAERSK MÉXICO, S.A. DE } \\
\text { C.V. }\end{array}$ \\
\hline SEALAND & $\begin{array}{l}\text { MAERSK MÉXICO, S.A. DE } \\
\text { C.V. }\end{array}$ \\
\hline $\begin{array}{l}\text { MEDITERRANEAN SHIP- } \\
\text { PING CO. S.A }\end{array}$ & $\begin{array}{l}\text { MEDITERRAEAN SHIPPING } \\
\text { CO. MÉXICO, S.A. DE C.V. }\end{array}$ \\
\hline GALBORG PTE. LTD & $\begin{array}{l}\text { NAVEMAR DE MÉXICO S.A. } \\
\text { DE C.V. }\end{array}$ \\
\hline NORDANA LINE & $\begin{array}{l}\text { NAVEMAR DE MÉXICO S.A. } \\
\text { DE C.V. }\end{array}$ \\
\hline TAINEON YUSE LINE, LTD & $\begin{array}{l}\text { NIPPON YUSEN KAISHAN- } \\
\text { YK LINE DE MÉXICO S. A. } \\
\text { DE C. V. }\end{array}$ \\
\hline SEABOARD MARINE & $\begin{array}{l}\text { ORIENT OVERSEAS CON- } \\
\text { TAINER LINES MÉXICO, S.A. } \\
\text { DE C.V. }\end{array}$ \\
SEAMARITIMA S.A. DE C.V \\
\hline UIEnte: ElabOIaCIOn
\end{tabular}

Fuente: Elaboración propia

Nótese que hay influencia de multinacionales en la gestión logística portuaria de México. Mientras que para el Puerto de Cartagena su vía principal es:

La Avenida Pedro de Heredia de 4 carriles dos de los cuales son para el solo bus de su Sistema Integrado de Transporte Masivo Transcaribe.

La Avenida Santander que comunica el Aeropuerto con la Zona Turística e histórica de la ciudad es una gran autopista que conecta de manera rápida con el sector hotelero turístico.

El Anillo vial para donde se sigue construyendo 
y ampliando la ciudad, comunica el barrio Crespo con la Zona Norte en donde se halla el Aeropuerto Internacional Rafael Núñez. El Vía ducto que pasa por arriba de la zona de manglares permitirá mayor movilidad a viajeros y evitar el paso por zonas de gran congestión en alta temporada.

El Corredor de Carga que une la Sociedad Portuaria Regional de Cartagena. el sector logístico portuario con el petroquímico de Mamonal.

Tabla 3: Las Agencias Marítimas y Líneas Navieras que ofrecen servicios de carga contenedorizada en el Puerto de Cartagena son:

\begin{tabular}{|c|c|}
\hline Líneas Navieras & Agencias Marítimas \\
\hline Alianca & HAMBURG SUD \\
\hline APL & ALTAMAR LTDA \\
\hline CMA CGM & Agencia Internacional Ltda. \\
\hline COSCO CONTAINER LINES & Oceánica Ltda. \\
\hline $\begin{array}{l}\text { EVER GREEN MARINE } \\
\text { CORP. }\end{array}$ & $\begin{array}{l}\text { Marítima del Caribe Internacio- } \\
\text { nal Ltda. }\end{array}$ \\
\hline HAMBURG SUD & HAMBURG SUD \\
\hline HAPAG-LLOYD & Aníbal Ochoa y Cia. \\
\hline INTERMARINE & Caribbean Shipping Agency \\
\hline K LINE & CARIBBSA \\
\hline KING OCEAN SERVICE & Cia Aduanera \\
\hline MAERSK & Global Shipping Agencies \\
\hline MARFRET & Maersk Colombia S.A. \\
\hline $\begin{array}{l}\text { MEDITERRANEAN SHIP- } \\
\text { PING CO S.A. }\end{array}$ & Eurocaribbean shipping \\
\hline $\begin{array}{l}\text { MELFI MARINE CORPORA- } \\
\text { TION }\end{array}$ & Frontier Agencia Marítima \\
\hline MITSUI OSK LINES & Roldán y Cia. \\
\hline NYK & Servinaves Ltda. \\
\hline SEA LAND & Transmares Agencia Marittima \\
\hline SEA BOARD MARINE & $\begin{array}{l}\text { Trasnportación Marítima Co- } \\
\text { lombiana }\end{array}$ \\
\hline YANG MING & Agencia Marítima Altamar Ltda. \\
\hline ZIM & Aviatur \\
\hline
\end{tabular}

Fuente: Elaboración propia

Es importante aclarar que en esa segunda tabla las Agencias Marítimas para el caso de Cartagena no coinciden con las Líneas Marítimas, aunque también nótese que hay coincidencias de Agencias y Navieras como consecuencia de la globalización en cuanto al a infraestructura vial es mucho mejor trazada la del Puerto de Veracruz debido a la extensión y diseño de la ciudad, no tiene muchos cuerpos de agua como el caso de Cartagena y se ha podido construir varios Muelles ubicados estratégicamente y se ha rellenado gran parte de las Zonas propias para actividades logísticas propias de un Puerto. Las condiciones morfológicas favorecen al Puerto de Veracruz, aunque las alianzas estratégicas del Puerto de Cartagena proyectan gran crecimiento en maniobras logísticas portuarias. Las ciudades de Cartagena y Veracruz, además de ser hermanas tienen la misma vocación logística portuaria, pero hay que reconocer debido a la gobernanza, es decir, el buen gobierno Veracruz es más fuerte en su crecimiento, nivel de bienestar, bajo nivel de desempleo, aunque Cartagena ha disminuido notoriamente durante el año 2018, ya que según el Dane está en $7.5 \%$ en el último trimestre, no obstante, se incluye la informalidad que es uno de los grandes retos del gobierno.

\section{Referencias bibliográficas}

Banco Interamericano de Desarrollo. BID. (2018) En Red de Observatorios Nacionales, tomado de http://logisticsportal.iadb.org/ node $/ 2024$.

Balza-Franco, V., \& Cardona, D. A. (2016). Economías de aglomeración empresarial y políticas públicas de competitividad desde un enfoque global hacia un contexto latinoamericano: Una revisión conceptual. Revista Espacios, 37(36), 16373601-16373635.

Barbero, J., Castro, L., Abad, J., \& Szenkman, P. (2011). Un transporte para la equidad y el crecimiento. Aportes para una estrategia nacional de movilidad y logística para la Argentina del Bicentenario. Documento de Trabajo, (79).

Cardona Arbeláez, D., Balza Franco, V., \& Henríquez Fuentes, G. (2017). Innovación en los procesos logísticos: Retos locales frente al desarrollo global.

Cardona, D., Rada, A. M., \& Palma, H. G. H. (2017). Creación de empresa como pilar para el desarrollo social e integral de la región caribe: Apuntes críticos. Saber, ciencia y libertad, 12(1), 15. 
Castell, O. C., Niño, E. D. S., Castellanos, R. S. U., \& Lugo, C. Y. (2017). Infraestructura portuaria en Colombia: asimetrías entre el puerto de Buenaventura y el puerto de Cartagena para el año 2015. Universidad \& Empresa, 19(32), 87-106.

Cipoletta Tomassian, G., Pérez, G., \& Sánchez, R. (2010). Políticas integradas de infraestructura, transporte y logística: experiencias internacionales y propuestas iniciales. CEPAL. http:// repositorio.cepal.org/handle/11362/6350

Coordinación General de Puertos (2018) Coordinación General de Puertos y Marina Mercante, recuperado el 2 de junio de 2018 en https:// www.gob.mx/puertosymarinamercante/articulos/nuevo-puerto-de-veracruz-sera-la-obra-portuaria-mas-importante-de-los-ultimos-100anos-en-mexico-sct-89346?idiom $=$ es

Domínguez Pérez, O. (Pág. 89, 1990). El Puerto de Veracruz: la modernización a finales de Siglo XIX. Recuperado en https://cdigital.uv.mx/bitstream/123456789/8520/2/anuaVII-pag87-102. pdf

Fuentes Campos, R. (2018). Estudio de soluciones para la ampliación del puerto de Veracruz (México). Estudio de soluciones del muelle norte de contenedores (Doctoral dissertation). Tomado de https://riunet.upv.es/handle/10251/94553

Fuentes Campos, R. (2018)2. Estudio de soluciones para la ampliación del puerto de Veracruz (México). Estudio de soluciones del muelle norte de contenedores (Doctoral dissertation). Tomado de https:/riunet.upv.es/bitstream/ handle/10251/94553/01_Memoria.pdf?sequen$\mathrm{ce}=1 \&$ isAllowed $=\mathrm{y}$

Fuentes, D. D. (2014). Transporte y Logística en La economía mundial. Revista de Economía Mundial, (38), 19-22. Huelva. España

González Lizcano, K. L. (2017). Evaluación de la implementación de tecnologías y certificaciones en construcción sostenible entre las ciudades de Sao Paulo, Brasil, y Bogotá, Colombia.
Trabajo de Grado. Universidad Católica de Colombia. Facultad de Ingeniería. Programa de Ingeniería Civil. Bogotá, Colombia

Gregory J. Brock, (2014) "The long run industrial growth of Veracruz state, 1955-2008", Journal of Economic Studies, Vol. 41 Issue: 6, pp.821-832, https://doi.org/10.1108/JES-022013-0020

Grupo Puerto de Cartagena (2017), Ciudad Puerto- sostenible. Boletín recuperado de https:// www.puertocartagena.com/es/sala-de-prensa/ noticias/ciudad-puerto-sostenible

Hernández, H. G., Cardona, D. A., \& Del Rio, J. L. (2017). Direccionamiento Estratégico: Proyección de la Innovación Tecnológica y Gestión Administrativa en las Pequeñas Empresas. Información tecnológica, 28(5), 15-22.

Hernández-Palma, H., Cardona-Arbelaez, D., \& Raad-Pardo, A. (2016). La innovación empresarial como impulsora de las Pymes en la Región Caribe. Revista Panorama EconóMico, 24, 239-248. Recuperado de http://revistas.unicartagena.edu.co/index.php/panoramaeconomico/ article/view/1559

Juan Gabriel Brida, Nicolás Garrido, María Jesús Such Devesa, (2012) “Cruise passengers' satisfaction: Cartagena de Indias", Benchmarking: An International Journal, Vol. 19 Issue: 1, pp.52-69, https://doi.org/10.1108/14635771211218353

Pitre-Redondo, R., Cardona-Arbeláez, D., \& Hernández-Palma, H. (2017). Proyección del emprendimiento indígena como mecanismo de competitividad en el postconflicto colombiano. Revista de Investigación, Desarrollo e Innovación, 7(2), 231-240.

López, J. A. (2018). La competitividad en el marco de políticas para ciudades sostenibles: Caso Cartagena, Colombia. InterNaciones, 5(13), 101-130.

Meisel-Roca, A. (Pág 3, 2015). Cartagena 19001950: a remolque de la economía nacional. Banco de la República de Colombia Documentos de Trabajo. 
Análisis comparativo de la logística sostenible entre el Puerto de Veracruz en México y el

Montoya, R. A. G., Espinal, A. A. C., \& Vahos, J. D. H. (2018). Modelo de centro de distribución verde: amigabilidad con el medio ambiente y eficiencia operacional usando un enfoque de procesos y un metaheurístico de búsqueda tabú. Revista Ingenierías Universidad de Medellín, 16(31), 199-217. http://hdl.handle. net/11407/4684

Peyrelongue, C. M. (2002) Puertos y Pivotes en México. Límites y posibilidades. México, Revista de la CEPAL 76 recuperada en https://repositorio.cepal.org/bitstream/handle/11362/10805/076123141_es.pdf?sequen$\mathrm{ce}=1$

Relación de Navieras y Contendores en Puertos Mexicanos (2018) recuperado el 5 de junio en: https:/www.gob.mx/cms/uploads/attachment/ file/161063/Relaci_n_Navieras_contenedores puertos_mexicanos.pdf

Rinaudo, C. (2012) Una mirada desde el puerto de Veracruz. Ed IRD. Biblioteca Veracruzana. Veracruz. México.

Toribio, J. M. F. (2010). Patrimonio territorial y desarrollo sostenible: un estudio comparativo en Iberoamérica y España. Estudios Geográficos, 71(268), 129-159.

Torres Tello, Giovanna Patricia (2018): la dinámica de las redes empresariales y la implementación de una estrategia de expansión territorial. In: dinámica económica y procesos de innovación en el desarrollo regional. Universidad Nacional Autónoma de México y Asociación Mexicana de Ciencias para el Desarrollo Regional A.C, Coeditores, México. ISBN UNAM: 978-607-30-0000-0, AMECIDER: 978-60796649-7-8 